\title{
A NOTE ON A THEOREM OF CHISWELL
}

\author{
STEPHEN JACKSON AND LUCA Q. ZAMBONI
}

(Communicated by Ronald M. Solomon)

\begin{abstract}
In this note we give an alternative proof of a theorem of I. M. Chiswell which states that every finitely generated group which acts non-trivially on a $\Lambda$-tree admits a non-trivial action on an $\mathbb{R}$-tree.
\end{abstract}

In [4], P. B. Shalen asked the following question: If a finitely generated group $G$ admits a non-trivial action without inversions on a $\Lambda$-tree, then does it also admit a non-trivial action without inversions on some $\mathbb{Z}$ - tree? Here $\Lambda$ denotes an arbitrary ordered abelian group. In [2], I. M. Chiswell showed that one can reduce the question to the case where $\Lambda=\mathbb{R}$, i.e., he proves the following theorem:

Theorem. Let $\Lambda$ be an ordered abelian group. Let $G$ be a finitely generated group admitting a non-trivial action (possibly with inversions) on a $\Lambda$-tree $T$. Then $G$ admits a non-trivial action on some $\mathbb{R}$ - tree.

Chiswell's proof of the theorem depends on the fact that every ordered abelian group can be embedded in some non-standard model of the first-order theory of the real numbers. In this sense, the proof is non-constructive. In fact, the mere existence of a non-standard model of $\mathbb{R}$ involves a non-constructive process. They may be constructed using the Compactness Theorem of first-order logic, which is a consequence of Gödel's Completeness Theorem (cf. [3] ), or by using a non-principal ultrafilter on an infinite set. Both of these methods in turn depend on some form of the axiom of choice. In this note, we give an alternative proof of the above theorem which is independent of the existence of a non-standard model for the real numbers. We begin by introducing some terminology. For a more comprehensive account of the basic theory of $\Lambda$-trees, we refer the reader to [1] and [4].

Let $G$ be a group acting (by isometries) on a $\Lambda$ - tree $T$. An element $g \in G$ is called an inversion (or phantom inversion), if $g^{2}$ has a fixed point but $g$ does not. If $g \in G$ is not an inversion, we denote the translation length of $g$ by $|g|$. It is defined to be the minimum of $d(x, g(x))$ as $x$ ranges over all points of $T$. Let $A_{g}$ denote the characteristic subtree of $g$, i.e., $A_{g}=\{x \in$

Received by the editors January 15, 1994.

1991 Mathematics Subject Classification. Primary 20E99, 06F20.

The first author was partially supported by NSF grant $90-07808$.

The second author was partially supported by a grant from the Texas Advanced Research Program and a Faculty Research Grant from UNT. 
$T|d(x, g(x))=| g \mid\}$. Then, $A_{g}$ is a non-empty closed subtree of $T$ invariant under the action of $g$. If $|g|=0$, then $A_{g}$ is merely the fixed point set of $g$, while if $|g|>0$, then $A_{g}$ is a linear tree on which $g$ acts by translation by $|g|$ (cf. [1], Theorem 6.6). If $g$ is an inversion, then by convention $|g|=0$ and $A_{g}=\varnothing$. Finally, the action of $G$ is said to be non-trivial if $|g|>0$ for some $g \in G$.

Proof (of the theorem). In case $\Lambda$ is archimedean, in which case it is orderpreserving isomorphic to a subgroup of $\mathbb{R}$, then the result of the theorem is immediate since $G$ acts non-trivially on the $\mathbb{R}$ - tree $\mathbb{R} \otimes_{\Lambda} T$ (cf. [1], Proposition 7.1). Thus, we can assume that $\Lambda$ is non-archimedean. We can further assume without loss of generality that the group $G$ acts without inversions. In fact, if $G$ acts non-trivially on a $\Lambda$ - tree $T$, then $G$ acts non-trivially and without inversions on the $\frac{1}{2} \Lambda-$ tree $\frac{1}{2} \Lambda \otimes_{\Lambda} T$ (cf. [1], Proposition 7.1).

Let $g_{1}, g_{2}, \ldots, g_{n}$ be generators for $G$ such that $A_{1} \cap A_{2} \cap \ldots \cap A_{n} \neq \varnothing$, where for each $1 \leq i \leq n, A_{i}$ denotes the charactersitic subtree of $g_{i}$ (cf. [2], Lemma 4). Assume that the generators $g_{1}, g_{2}, \ldots, g_{n}$ are ordered so that $0 \leq\left|g_{1}\right| \leq\left|g_{2}\right| \leq \ldots \leq\left|g_{n}\right|$. By hypothesis, $\left|g_{n}\right| \neq 0$. Let

$$
\Lambda^{\prime}=\left\{\lambda \in \Lambda \mid \exists m\left(m \in \mathbb{Z} \text { and } m\left|g_{n}\right|>\lambda\right)\right\} .
$$

Let $x \in A_{1} \cap A_{2} \cap \ldots \cap A_{n}$, and define $L_{x}: G \rightarrow \Lambda$ by $L_{x}(g)=d(x, g(x))$ for all $g \in G$. Then, $L_{x}$ is a Lyndon length function with values in the ordered abelian subgroup $\Lambda^{\prime}$ (cf. [1], Definition 5.1 and Example 5.3). To see that $L_{x}$ actually takes values in $\Lambda^{\prime}$, we first note that $L_{x}$ satisfies the triangle inequality since $L_{x}(g h)=d(x, g h(x)) \leq d(x, g(x))+d(g(x), g h(x))=d(x, g(x))+$ $d(x, h(x))=L_{x}(g)+L_{x}(h)$. Thus, if $g \in G$ is a word of length $m$ in the generators $g_{1}, g_{2}, \ldots, g_{n}$, then $L_{x}(g) \leq m L_{x}\left(g_{n}\right)=m\left|g_{n}\right|$. It follows that there exists a $\Lambda^{\prime}$-tree $T^{\prime}$ and an action of $G$ by isometries on $T^{\prime}$. In fact, $T^{\prime}$ is identified with the subtree of $T$ spanned by the orbit $G x$ and the action of $G$ on $T^{\prime}$ is simply by restriction (cf. [1], Theorem 5.4). Moreover, since $x \in A_{1} \cap A_{2} \cap \ldots \cap A_{n}$, the translation length function with respect to the action of $G$ on $T^{\prime}$ coincides with that of the action of $G$ on $T$.

Let

$$
M=\left\{\lambda \in \Lambda^{\prime} \mid \forall m\left(m \in \mathbb{Z} \Rightarrow\left|g_{n}\right|>m \lambda\right)\right\} .
$$

Then $M$ is a maximal convex subgroup of $\Lambda^{\prime}$ and the quotient $\Lambda^{\prime} / M$ is an archimedean ordered abelian group. In fact, let $\left[\lambda_{1}\right]$ and $\left[\lambda_{2}\right]$ be in $\Lambda^{\prime} / M$ with $\left[\lambda_{1}\right]>0$. Then there exists an integer $m_{1}$ and a representative $\lambda_{1}$ of $\left[\lambda_{1}\right]$ such that $m_{1} \lambda_{1}>\left|g_{n}\right|$. Similarly, there exists an integer $m_{2}$ and a representative $\lambda_{2}$ of $\left[\lambda_{2}\right]$ such that $m_{2}\left|g_{n}\right|>\lambda_{2}$. Thus, $m_{1} m_{2} \lambda_{1}>\lambda_{2}$.

Let $h_{1}: \Lambda^{\prime} \rightarrow \Lambda^{\prime} / M$ be the canonical homomorphism, and let $h_{2}: \Lambda^{\prime} / M \rightarrow$ $\mathbb{R}$ be an order-preserving group homomorphism of $\Lambda^{\prime} / M$ onto an additive subgroup of $\mathbb{R}$. Then, the composition $h=h_{2} \circ h_{1}: \Lambda^{\prime} \rightarrow \mathbb{R}$ is a homomorphism of ordered abelian groups with the property that if $\lambda \geq 0$ in $\Lambda^{\prime}$, then $h(\lambda) \geq 0$ in $\mathbb{R}$. Let $T_{\mathbb{R}}$ denote the $\mathbb{R}-$ tree $\mathbb{R} \otimes_{\Lambda^{\prime}} T^{\prime}$ (cf. [1] Proposition 4.4). Then, $G$ acts non-trivially on $T_{\mathbb{R}}$. In fact, the translation length of $g_{n}$ with respect to the induced action of $G$ on $T_{\mathbb{R}}$ is equal to $h\left(\left|g_{n}\right|\right)$, which in turn is different from zero since $\left[\left|g_{n}\right|\right]>0$ in $\Lambda^{\prime} / M$.

We end by noting that in our proof as well as in Chiswell's proof of the above theorem, "freeness" is not preserved, even under those circumstances in which 
it is theoretically possible. In fact, any element $g \in G$ whose translation length is in $M$ will have a fixed point when viewed as an isometry of the $\mathbb{R}$-tree $T_{\mathbf{R}}$. For instance, let $\Lambda=\mathbb{Z} \oplus \mathbb{Z}$ (with lexicographic order), and let $G$ denote the free abelian group on two generators $g_{1}$ and $g_{2}$. Then $G$ acts freely on the $\Lambda$ - tree $T=\Lambda$ by translation. Let $\left|g_{1}\right|=(0,1)$ and $\left|g_{2}\right|=(1,0)$. Then, since the translation length of $g_{1}$ is infinitessimal with respect to that of $g_{2}$, we obtain that $g_{1}$ will fix each point of the $\mathbb{R}$ - tree $T_{\mathbb{R}}$, which in this case is simply $\mathbb{R}$. Yet, in this case $G$ admits a free action on $\mathbb{R}$ by translations; e.g., $\left|g_{1}\right|=1$, and $\left|g_{2}\right|=\sqrt{2}$.

\section{REFERENCES}

1. R. Alperin and H. Bass, Length functions of group actions on $\Lambda$-trees, Combinatorial Group Theory and Topology, Ann. of Math. Stud., no. 3, Princeton Univ. Press, Princeton, NJ, 1987, pp. 265-378.

2. I. M. Chiswell, Non-trivial group actions on $\Lambda$-trees, Bull. London Math. Soc. 24 (1992), 277-280.

3. H. Enderton, A mathematical introduction to logic, Academic Press, New York, 1972.

4. P. B. Shalen, Dendrology of groups: an introduction, Essays in Group Theory (S. M. Gersten, ed.), Math. Sci. Res. Inst. Publ., vol. 8, Springer, New York, 1987, pp. 265-319.

Department of Mathematics, The University of North Texas, Denton, Texas 76201

E-mail address: jackson@sol.acs.unt.edu

E-mail address: fg70@vaxb.acs.unt.edu 